\title{
New technology and the changing world of cancer
}

\author{
Peter T Scardino
}

Cancer is the leading cause of death below the age of 85 years in most developed countries. Whilst the mortality rate has been falling for the past 10 years, the overall age-specific risk of dying from cancer remains as high as it was 50 years ago. Cancer is an age-related disease and our population is aging rapidly, so the number of new cases will grow substantially.

Technologic advances and research efforts are profoundly changing our understanding of cancer and our ability to treat it effectively. This was dramatically apparent at the National Cancer Institute Specialized Programs of Research Excellence (SPORE) Investigators Workshop held in Washington, DC in July 2005. Attended by over 1,000 scientists and physicians, the workshop covered the most recent advances in translational research.

Early detection has led to improved survival rates for many cancers, especially tumors of the prostate, breast and colon. Ironically, detection is so effective that the risk of unnecessary treatment has become a real problem. The quality of imaging with CT and MRI has greatly improved, and molecular imaging is rapidly progressing. In a remarkable study presented at the meeting, radiolabeled dihydrotestosterone was used with positron emission tomography to visualize the androgen receptor in metastatic lesions of hormone-refractory prostate cancer. Following therapy specifically targeting the receptor, dihydrotestosterone uptake in the lesions diminished, confirming destruction of the molecular target. Similar results were achieved with trastuzumab targeting of the HER2 receptor.

In another study, 'global' RNA interference was used to identify vulnerable genetic targets in cancers. The goal was to identify pathways that can be targeted to kill cells with key genetic defects. Delivering systemic cancer therapy tailored to specific genetic defects is no futuristic dream. Several teams of investigators have reported dramatic responses to gefitinib (an inhibitor of the epidermal growth factor
....50 years of

basic scientific

research in

molecular

biology are

bearing fruit... .

cancer care

is undergoing

a quiet, but

dramatic,

revolution.

PT Scardino is Editorin-Chief of Nature

Clinical Practice

Urology.

\section{Competing interests}

The author declared he has no competing interests.

www.nature.com/clinicalpractice doi:10.1038/ncpuro0298 receptor) in patients with non-small-cell lung cancer who exhibit specific mutations in the epidermal growth factor receptor gene. Less than $10 \%$ of patients have such mutations and in these individuals the response rates are profound. While clinical trials show statistically significant survival advantages for many biologic agents, the overall benefit is usually only a matter of weeks. In the future, the expense of these agents will surely be justified as research focuses on defining the molecular characteristics of the uncommon tumors that respond to them.

Our ability to characterize cancers will change dramatically with the widespread use of molecular diagnostics and the introduction of systems pathology. Immunohistochemical analysis of tissue specimens is now an essential element of a modern surgical-pathology laboratory. Molecular analysis can already characterize the major types of renal-cortical tumors, differentiating aggressive tumors from benign lesions. Diagnosis by percutaneous biopsy with molecular genetic characterization, will soon be coupled with percutaneous treatment of the growing number of small renal masses found incidentally on imaging studies. Tumors that are unsuitable for percutaneous treatment will be treated with minimally invasive laparoscopic partial nephrectomy or open "mini-lap" excision of small renal masses. Image-guided therapy will further change oncology, allowing surgical oncologists and interventional radiologists to guide diagnostic needles for biopsy and therapeutic instruments or energy for the obliteration of tumors.

Finally, 50 years of basic scientific research in molecular biology are bearing fruit. This progress is matched by the technological wizardry of imaging and minimally invasive therapy. Cancer care is undergoing a quiet, but dramatic, revolution. One could only come away from the SPORE Investigators Workshop optimistic about the future prospects for patients with cancer. 